\title{
Eisenmenger syndrome and pericardial effusion: the challenge of diagnosing "tamponade" in complex congenital heart disease
}

\begin{abstract}
Cardiac tamponade is a life-threatening condition wherein overwhelming intrapericardial pressure from a either rapidly progressive or a sizeable pericardial effusion leads to collapse of the cardiac chambers, impairment in diastolic filling and hemodynamic compromise. Clinical and echocardiographic signs, such as a pulsusparadoxus, significant transmitral inflow variations, and visible chamber collapse aid in determining the need for emergent Pericardiocentesis. We describe a case where a hemodynamically significant pericardial effusion warranting emergent Pericardiocentesis, presents with none of the traditional signs of tamponade. It is first written case report of a very large pericardial effusion in the setting of Eisenmenger syndrome where worsening dyspnea, hypoxia and cyanosis secondary to right-to-left shunting was the clinical correlate of cardiac tamponade necessitating emergent Pericardiocentesis.
\end{abstract}

Volume I Issue 4 - 2014

\author{
Margaret Cases, Daniel Patton, Amer Johri \\ Kingston General Hospital, Canada
}

\begin{abstract}
Correspondence: Mahmut Yesin, Department of Cardiology, Kosuyolu Kartal Heart Training and Research Hospital, Denizer Cad. Cevizli, Kvs. No: 2 Cevizli Kartal /Istanbul,Turkey, Tel 902165001599,Email margaret.r.b.cases@queensu.ca
\end{abstract}

Received: July 27, 2014 | Published: September 30, 2014
Abbreviations: AVSD, atrioventricular septal defect; CTPA, $\mathrm{CT}$ pulmonary angiogram; RA, right atrial; RV, right ventricular

\section{Case report}

A 31year-old female with Trisomy21, uncorrected complete atrioventricular septal defect (AVSD) and consequent Eisenmenger syndrome presented to our emergency department with dyspnea, hypoxia, and worsening cyanosis. She was found to be hemodynamically stable with a heart rate of $70 \mathrm{bpm}$ and blood pressure of $100 / 60$. There was no pulsusparadoxus. She was notably cyanotic, platypneic, and hypoxic. Her oxygen saturation was between $80-85 \%$ despite $\mathrm{O}_{2}$ supplementation. Her recorded saturation at a clinic visit a year ago was $93 \%$ on room air. An arterial blood gas showed a $\mathrm{PaO} 2$ was $51 \mathrm{mmHg}$ on a $100 \% \mathrm{O}_{2}$. Her $\mathrm{pH}, \mathrm{PCO} 2$ and $\mathrm{HCO}^{3}$ were normal. She underwent an urgent $\mathrm{CT}$ pulmonary angiogram (CTPA), which confirmed features of Eisenmenger syndrome (i.e., complete AVSD, right ventricular hypertrophy, right atrial dilatation and an enlarged pulmonary artery) but showed no pulmonary embolus. The CTPA was negative for signs of pulmonary congestion or edema. Incidentally, a very large pericardial effusion was found. Additional history revealed that her functional capacity had steadily declined over the course of two weeks with marked dyspnea on exertion of less than a meter of walking.

An urgent echocardiogram confirmed the presence of a very large circumferential pericardial effusion with a maximum dimension of $40 \mathrm{~mm}$. There were no echocardiographic features of cardiac tamponade i.e., right atrial (RA) or right ventricular (RV) collapse, significant variations in mitral and tricuspid inflow velocities. The right ventricle was severely thickened $(18 \mathrm{~mm})$ but visually normal in function. Quantitation of RV function (such as TAPSE, or fractional shortening), shunting across the AVSD, and pulmonary pressures could not be obtained due limited echocardiographic windows as the patient was in acute distress. However, as was measured from a previous echocardiogram, her pulmonary arterial systolic pressure was at least $110 \mathrm{mmHg}$. Without having the traditional clinical or echocardiographic markers of tamponade, the challenge was delineating whether her presentation was due to severe pulmonary hypertension (i.e., end- stage Eisenmenger syndrome) or secondary to the large pericardial effusion, indicating a need for emergent Pericardiocentesis. Given the patient's respiratory distress and hypoxemia, which was considered a correlate of classic tamponade, an urgent Pericardiocentesis was performed. This resulted in an immediate and sustained improvement in her symptoms and oxygenation. She was transferred to the coronary care unit for ongoing management.

\section{Discussion}

Eisenmenger syndrome is a complication of systemic to pulmonary shunting. Pulmonary hypertension develops from chronic exposure of the pulmonary arterial bed to systemic pressures leading to RA dilatation and RV hypertrophy. Eventually, the existing shunt becomes bidirectional or reversed. This manifests as hypoxia, platypneic and cyanosis. In contrast to other conditions that cause chronic right ventricular pressure overload, Eisenmenger syndrome has a good long-term prognosis. Survival is $80 \%$ at 10 years. ${ }^{1}$ Progression to right-sided heart failure is relatively slow and may be attributed to a more resistant right ventricle. However, once it develops, it is a strong predictor of death. ${ }^{2}$ Pericardial effusion is common in Eisenmenger syndrome, and although it is a manifestation of heart failure, this has not been shown to be of prognostic significance. ${ }^{3}$ Cardiac tamponadeoccurs when over whelming intrapericardial pressure from either a rapidly expanding or sizeable pericardial effusion leads to collapse of the cardiac chambers and impairment in diastolic filling. The associated clinical and echocardiographic signs result from the exaggerated shifting of the interventricular septum towards the left ventricle as the compressed RV fails to accommodate the increased venous return during inspiration. This leads to a reduction in left ventricular size and stroke volume. ${ }^{4-6}$

To date, no existing literature characterizes clinical or echocardiographic "tamponade" in Eisenmenger syndrome. This case illustrates the difficulty in recognizing the perturbation in hemodynamics caused by the presence of a pericardial effusion in the setting of complex congenital cardiac diseases, even when aided by echocardiography. Two components of this patient's structural heart disease contributed to this: 
a) First, the absence of an intact septum eliminated the effects of ventricular interdependence on stroke volume. Instead, the unimpeded flow through the AVSD allowed for more rightto-left shunting and resulted in worsening of the patient's hypoxemia. This type of acute right-to-left shunting (or RLIAS) leading to acute hypoxia has been described in a patient with an atrial septal defect who developed tamponade. In addition, tamponade without a significant pulsusparadoxus or respiratory mitral inflow variation has been also been described in cases of atrial septal defects. ${ }^{7-9}$

b) Second, as with primary pulmonary hypertension, markedly elevated pulmonary pressures, non-compliance of the pulmonary vasculature and right-sided chambers, and right ventricular hypertrophy in Eisenmenger's syndrome, protects against diastolic chamber collapse in the face of a significant pericardial effusion. ${ }^{10,11}$ Therefore, chamber collapse will likely be a very late finding (Figure 1).
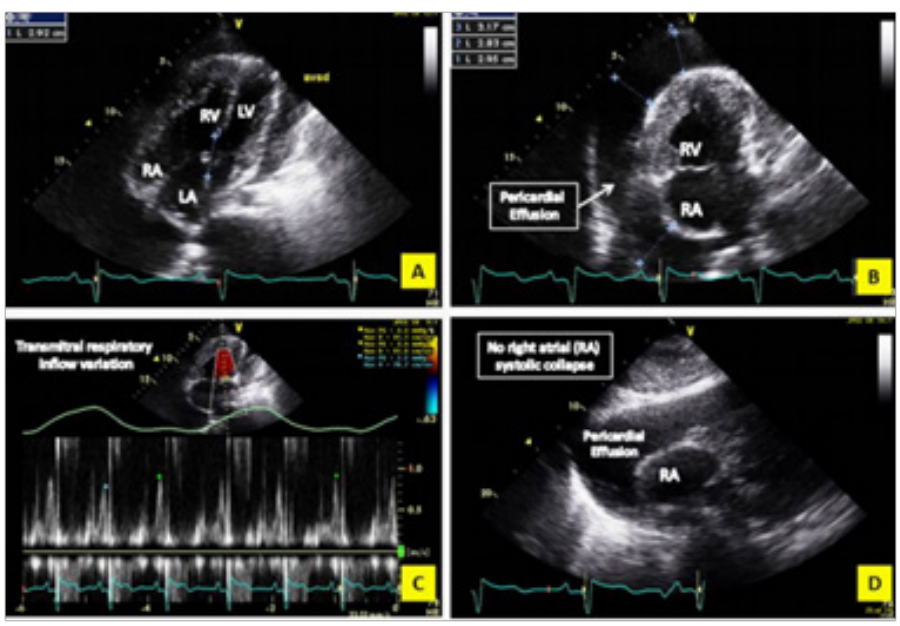

Figure I (A) Features of Eisenmenger Syndrome-shunt (AVSD), right Ventricular hypertrophy secondary to severe pulmonary hypertension (B) Large circumferential pericardial effusion (C) Absence of significant transmitral inflow variation with respiration (D) Absence of right atrial collapse.

\section{Conclusion and implications in clinical practice}

The prevalence of adult survivors of congenital heart disease is on the rise. Assessment of pericardial effusion and tamponade is a common scenario in a cardiology practice. However, because of the structural changes in complex congenital heart diseases, a hemodynamically significant pericardial effusion will not to manifest the classic signs of tamponade. This case illustrates how septal defects modify the effects of ventricular interdependence on stroke volume, resulting in the absence of a pulsusparadoxus and its associated traditional echocardiographic findings. The only clue to what is classically recognized as "tamponade" may be hypoxia as a result of increased right-to-left shunting due to non- compartmentalized diastolic restriction. Furthermore, this case shows how the development of pulmonary hypertension and the consequent adaptive atrial and ventricular changes in Eisenmenger syndrome can be protective against increasing intrapericardial pressure, preventing chamber collapse and hemodynamic compromise.

\section{Acknowledgement}

None.

\section{Conflicts of interest}

Authors declare that there is no conflict of interest.

\section{Funding}

None.

\section{References}

1. Haddad F, Doyle R, Murphy DJ,et al. Right ventricular function in cardiovascular disease, part II pathophysiology, clinical importance, and management of right ventricular failure. Circulation. 2008;117(13):17171731.

2. Daliento L, Somerville J, Presbitero P, et al. Eisenmenger syndrome. Factors relating to deterioration and death. Eur Heart J. 1988;19(12):18451855 .

3. Moceri P, Dimopoulos K, Liodakis E, et al. Echocardiographic predictors of outcome in eisenmenger syndrome. Circulation. 2012;126(12):14611468 .

4. Spodick DH. Acute cardiac tamponade. N Engl J Med. 2003;49(7):684690.

5. Maisch B, Seferovic PM, Ristic AD, et al. Guidelines on the diagnosis and management of pericardial diseases executive summary; The Task force on the diagnosis and management of pericardial diseases of the European society of cardiology. Eur Heart J. 2004;25(7):587-610.

6. Otto CM, Pearlman AS. Textbook of clinical echocardiography. USA: W.B. Saunders Company; 1955. p. 404.

7. Winer HE, Kronzon I. Absence of paradoxical pulse in patients with cardiac tamponade and atrial septal defects. Am J Cardiol. 1979;44(2):378-380.

8. Marples IL, Heap MJ, Suvarna SK, et al. Acute right-to-left interatrial shunt; an important cause of profound hypoxia. $\mathrm{Br} J$ Anaesth. 2000;85(6):921-925.

9. Solar M, Fridrich J, Ceral J, et al. Acute hypoxia due to right to left blood shunting in a patient with atrial septal defect. Resuscitation. 2007;74(2):382-385.

10. Frey MJ, Berko B, Palevsky H, et al. Recognition of cardiac tamponade in the presence of severe pulmonary hypertension. Ann Intern Med. 1989;111(7):615-617.

11. Shabetai R. Changing concepts of cardiac tamponade. J Am Coll Cardiol. 1988;12(1):194-195. 